\title{
NEW RESULTS FOR TEACHING SHIP HANDLING USING FAST TIME SIMULATION
}

\author{
Knud Benedict ${ }^{1)}$, Michèle Schaub ${ }^{1)}$, \\ Michael Baldauf ${ }^{1)}$, Michael Gluch ${ }^{1)}$, \\ Matthias Kirchhoff'), Caspar Krüger ${ }^{2)}$ \\ ${ }^{1)}$ Department of Maritime Studies Warnemuende / Institute ISSIMS \\ Hochschule Wismar, University of Applied Sciences - Technology, \\ Business and Design (Germany) \\ ${ }^{2)}$ ISSIMS GmbH (Germany)
}

\begin{abstract}
Some years ago, the SAMMON software tool box was introduced for "simulation augmented manoeuvring design, monitoring \& conning". It is based on the innovative "rapid advanced prediction \& interface technology" (RAPIT) to simulate the ships motion by fast time simulation FTS with complex dynamic math models and to display the ships track immediately in an electronic sea chart for any rudder, thruster or engine manoeuvre planned by the navigator. specifically, the SAMMON planning tool will be shown in its opportunities for achieving new knowledge for teaching of ships dynamic and training of ship handling elements. That system represents the full information from ships' manoeuvring documentation and from additional trial results squeezed in a ship dynamic model, capable of simulating environmental effects by using the RAPIT technology. The paper introduces the variety of opportunities of the training tools by presenting use case studies, e.g. For drifting under wind and how to get simple formulas for drift speed related to wind speed. Additionally, two complex manoeuvring strategies for a port arrival scenario will be compared for a ship with azimuth propeller to find out potential alternatives with less fuel consumptions and emissions.
\end{abstract}

Keywords: fast-time manoeuvring simulation; voyage planning; dynamic prediction methods; simulator training

\section{Introduction and concept for fast-time simulation}

\section{Fast-Time Simulation Software to be used Simulation Support}

According to the IMO standards for voyage planning from berth-to-berth there is a need to prepare harbour approaches in a new quality with complete berthing plans specifically in companies with high safety standards like cruise liners. These 
plans are helpful in briefing procedures to agree on a concept within the bridge team and also for the discussion and briefing with the pilot. But still the plan for the potential manoeuvres could be developed in a contemplative way, only by "thinking ahead". With the unique SAMMON software an innovative electronic tool is now available to provide support for planning those manoeuvring concepts by Fast Time Simulation FTS.

It will improve also the Ship Handling simulator training which has proven high effect. However, it is based on real-time simulation, and i.e. $1 \mathrm{sec}$ calculation time by the computers represents one second manoeuvring time as in the real world. This is a very time-consuming process.

In the near future the method of Fast-Time Simulation FTS will be used for increasing the effectiveness of training and also the safety and efficiency of manoeuvring real ships.

That system represents the full information from Ships' manoeuvring documentation and additional trial measurements, which have been condensed in a complex ship dynamic simulation model, capable of simulating environmental effects by using the innovative "Rapid Advanced Prediction \& Interface Technology" (RAPIT). Even with standard computers it simulates 1000 times faster than real time: in 1 second computing time it manages to simulate a manoeuvre lasting up to 20 minutes. This technology was initiated in research activities of the "Institute for Innovative Ship Simulation and Maritime Systems" ISSIMS at the Maritime Simulation Centre Warnemuende MSCW, which is a part of Hochschule Wismar, University of Applied Sciences - Technology, Business \& Design in Germany, specifically in its Department of Maritime Studies. The technology has been further developed by the start-up company ISSIMS GmbH ${ }^{1}$.

There are several modules of the FTS simulation system:

In the centre stands SAMMON as the innovative system for "Simulation Augmented Manoeuvring - Design, Monitoring \& Conning". It comprises several software modules, the two most important are (a) the Manoeuvring Design \& Planning Module and (b) the Manoeuvring Monitoring \& Conning Module with Multiple Dynamic Manoeuvring Prediction.

These modules are created both for lecturing and simulator training for ship handling and also to assist manoeuvring of real ships on-board, e.g. to prepare manoeuvring plans for challenging harbour approaches / departures.

Important tools are made to support the SAMMON, e.g. the SIMOPT software for modifying ship math model parameters both for simulator ships in SHS and for on board application of the SAMMON System and the SIMDAT software module for analysing / displaying simulation results both from simulations in SHS or SIMOPT /SAMMON and from real ship trials ${ }^{1)}$. 


\section{Application of SAMMON in the EU Project EURO-ZA and Objectives}

Also, in ongoing research projects the SAMMON tools are being used, e.g. in the ERASMUS+ project EURO-ZA. Several partner universities are working together:

from Europe: HS Wismar /Germany, SOLENT Southampton /UK and SAMK Rauma, Finland) and

from South Africa: DUT Durban, CPUT Cape Town and NMU Port Elizabeth as project co-ordinator.

The aim is a detailed analysis of the curriculums and facilities to evaluate similarities, differences and opportunities for improvement for all partners. The contribution of the ISSIMS institute of HS Wismar addresses the use of SAMMON FTS methods for ship handling training as one element of the potential improvement of training.

In this paper some findings from the use of the SAMMON Planning tool will be described, e.g. how to discuss and calculate the drift of ships under wind. Also, the first estimations will be presented to use SAMMON to compare the fuel consumption for different manoeuvring strategies. Additionally, a lot of samples for using SAMMON to explain ships dynamic were made to be seen on YouTube ${ }^{2}$. Other samples and details were presented at various conferences (Benedict et al. 2015; Benedict et al. 2016; Schaub et al. 2019; Benedict et al. 2021).

Familiarisation with Fast-Time Simulation interface and Example of Wind drift Interface of SAMMON Planning Tool

The interface of the SAMMON Manoeuvring Design \& Planning tool combines the following three windows (see Figure 1):

- The right window represents the steering / control panel: this is for adjusting the controls for the selected actual Manoeuvring Point MP (actual position in red) or entering the environment conditions e.g. wind, current and water depths (bottom right),

- The centre window displays the electronic navigational chart (ENC) where the simulated ship's motion is visualised: the ships positions are shown as black contours indicating in time intervals for the display range. The reference position can be shifted by means of the time slider at the bottom to any position of the already predicted track. There a new MP can be set and controls may be changed there,

- The left and top window display the ship status at the reference ship position on the track, indicated as ship shape in blue colour in the ENC - this status is defined by e.g. the current navigation data and actual ship manoeuvring control data. 


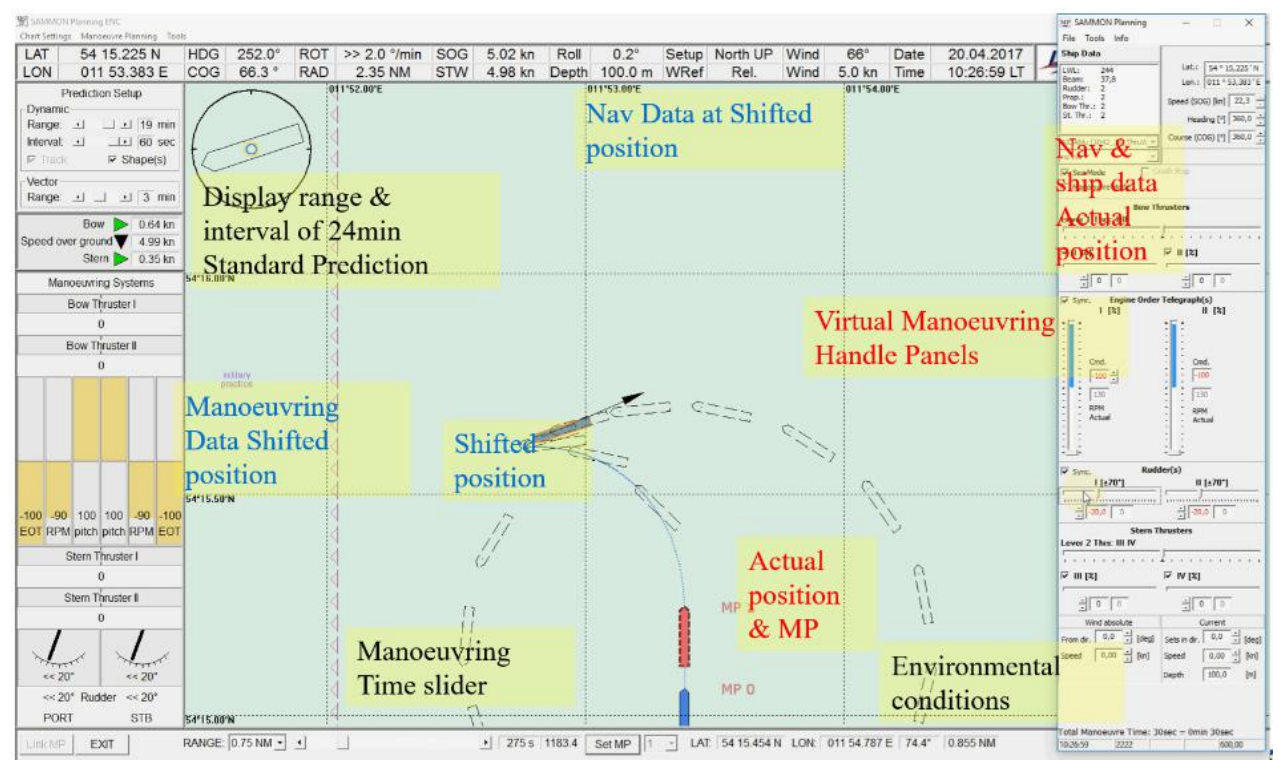

Figure 1. Interface elements of SAMMON Manoeuvring Design \& Planning tool with additional comments ${ }^{2}$ (more information under YouTube)

\section{Simulation and Formulas for pure Wind Drift Motion}

In the next figures the motion of a ship is discussed under wind speed $30 \mathrm{kn}$ from North $0^{\circ}$. The sample ship is a cruise liner of $253 \mathrm{~m}$ in length which is not using her propulsion. In Figure 2. the ship starts drifting from stop with zero speed. Each shape represents her position after 1 minute, the full drifting speed is reached very fast to be $\mathrm{SOG}=2.25 \mathrm{kn}$ after 1 or 2 minutes (this is indicated by equal distances of the shapes). The bow falls slightly down with the wind because she has the centre of wind area ahead of midships position. The drifting speed can be reduced by the thrusters: the ship would drift only with $\mathrm{SOG}=1.51 \mathrm{kn}$ if thrusters are used fully against the wind.

It might be very helpful to estimate the drift speed: The drift speed is nearly linear increasing with the wind speed, as the lower diagram proves. As a consequence, only one result would be enough (maybe better two or more because of potential bias...) to estimate a constant factor to estimate the drift for other wind speeds. 


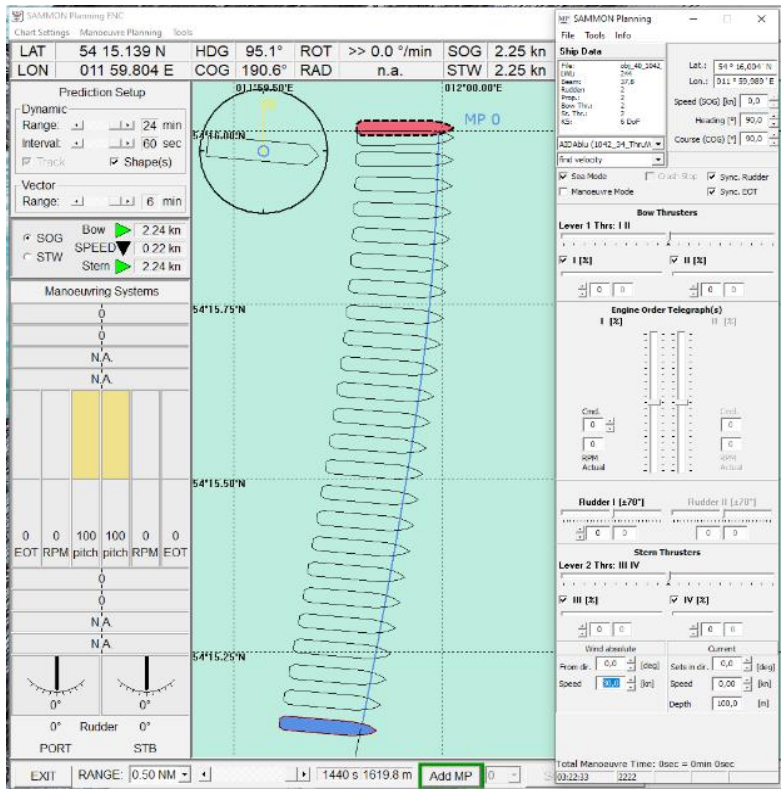

Drift Speed of Ship versus Wind Speed

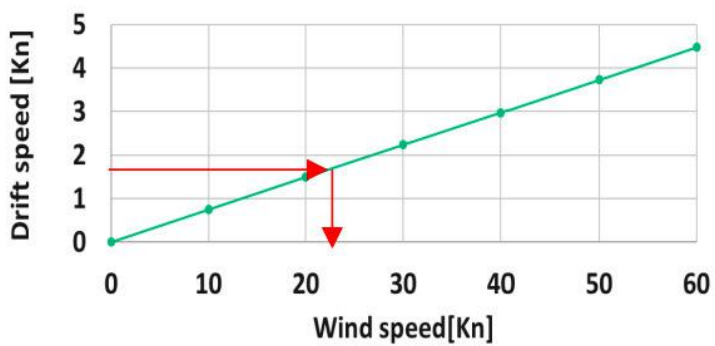

Figure 2. Transverse Drifting under Wind speed VA=30kn

(Cruise ship AIDAblu)

- Top: situation with drift speed $\mathrm{SOG}=2.25 \mathrm{kn}, \mathrm{HDG} 94^{\circ}, \mathrm{COG} 184^{\circ}$

- Below: diagram for drift speed versus wind speed with arrows indicating maximum drift balance by thrusters

For instance, in this sample the linear drift speed factor CD is about 0.075 , i.e. the drift speed is $7.5 \%$ of the wind speed. As a conclusion this can be used a suitable "Rule of Thumb) to estimate the drift speed e.g. for cruise ships for a given wind speed! In which way the drift direction can be changed by using the thrusters was discussed already in (Benedict et al. 2021). 


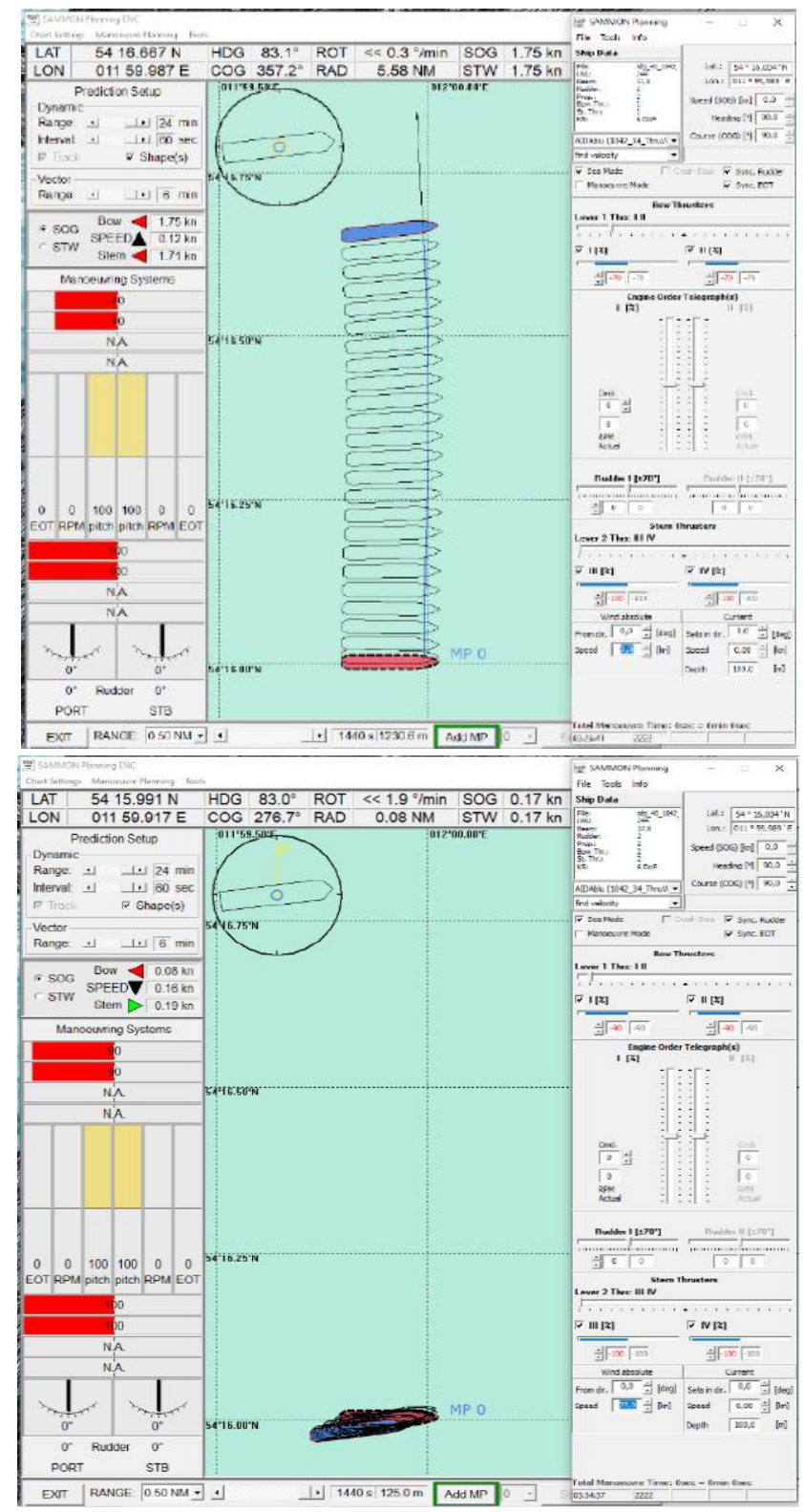

Figure 3. Experiment for Maximum Drift speed for full thruster with no wind: Result $1.75 \mathrm{kn}$. (Top) and test scenario to balance wind of $23 \mathrm{kn}$ by thruster (below) 
It is also important to know the absolute limit wind speed VA_lim which can possibly be balanced by thrusters which can be estimated by measuring the Maximum Drift speed VD_max for full thrusters with no wind (see Figure 3.). The result of 1.75 $\mathrm{kn}$ can be seen in the diagram as red horizontal line, as a result the Limit Wind speed can be seen as $23 \mathrm{kn}$ from the vertical arrow. Instead it is also possible to use a formula:

$$
\begin{aligned}
& V_{A_{-} \text {lim }}[\mathrm{kn}]=\frac{V_{D_{-} \max }}{C_{D}} \\
& V_{A_{-} \text {lim }}[\mathrm{kn}]=\frac{1.75}{0.075}=23 \mathrm{kn}
\end{aligned}
$$

\section{Simulation for drift balance making headway under Wind}

To keep the ship on straight track under Wind, normally rudder and drift angle are required to balance and steer the ship straight under beam wind. In the SAMMON planning module it can be easily investigated in a very short time which rudder and drift angle are generally needed for different wind conditions and ship's speeds: In Figure 4. several rudder angles were applied and observed to determine which drift angle on straight tracks with the respective courses had the ships found as balanced equilibrium conditions.

On the left side the results are shown for a ship with windward turning tendency, which has normally the centre of wind area aft behind COG. Therefore, e.g. with rudder amidships $0^{\circ}$ the ship turns into the wind and with increasing starboard rudder she finds other equilibriums on different straight tracks and courses out of the wind.

On the right side a ship with leeward turning tendency is shown with the centre of wind area fore of COG: for increasing starboard rudder she turns further out of the wind and finds an equilibrium with increasing COG. For rudder angles larger than $25^{\circ}$ the rudder effectiveness is decreasing to be seen that for $30^{\circ}$ and $35^{\circ}$ the ship does not turn anymore further and therefore cannot be brought out of the wind.

For ship b) on the right side the results for rudder and drift angle are plotted versus the absolute wind encounter angle and for two wind speeds in Figure 5 where the rudder and drift angle are plotted versus the absolute wind encounter angle between wind direction and ship's course (per definition $0^{\circ}$ for heading against the wind and $180^{\circ}$ for stern wind). The wind impact changes with the encounter angle and the speed ratio $\mathrm{VR}^{2} / \mathrm{V}^{2}$.

Such a diagram as in Figure 5 is the basis for understanding the wind effect and for estimating the required rudder and drift angle for route planning using the absolute wind speed VA and the constant speed V0 of the ship for a given EOT without wind together with the absolute wind encounter angle.

Apart from these data from simulation experiments or from measurements onboard ships, there is also the following simplified approach to calculate drift angles 
under wind making headway, using the value estimated for the constant wind drift factor CD under beam wind as in formula (1):

$$
\begin{aligned}
& \text { Best }=\arctan \left(\frac{V_{D} * \sin \propto_{A}}{V_{S}}\right) \\
& =\arctan \left(\frac{C D *\left(V_{A}+V S * \cos \propto_{A}\right) * \sin \propto_{A}}{V_{S}}\right)
\end{aligned}
$$

In this formula VA is the absolute wind speed and $\alpha \mathrm{A}$ is the absolute encounter angle between COG and wind direction, VS is the ship speed. Here is a sample for calculation of Required Drift angle from simplified approach (2) for the following sample conditions:

- Encounter angle: $\mathrm{COG} 161^{\circ}$ - Wind $061^{\circ}$, i.e. $\alpha \mathrm{A}=100^{\circ}$

- Speed ratio $\mathrm{VA}=30 \mathrm{kn}, \mathrm{VS}=5 \mathrm{kn}$, i.e. $\mathrm{VA} / \mathrm{VS}=6.0$

$$
\begin{aligned}
& \text { Best }==\arctan \left(\frac{0.075 *(30+5 * \cos 100) * \sin 100}{5}\right) \\
& \text { Best }=23,3^{\circ}
\end{aligned}
$$

This formula has an acceptable accuracy as "Rule of a Thumb"; in most cases it might present results a little too high (see dotted lines in) because lift forces at hull $\&$ rudder when making headway are not sufficiently considered.

Further results on wind impact, specifically on Turning Motion have been explained in detail in (Benedict et al. 2015).

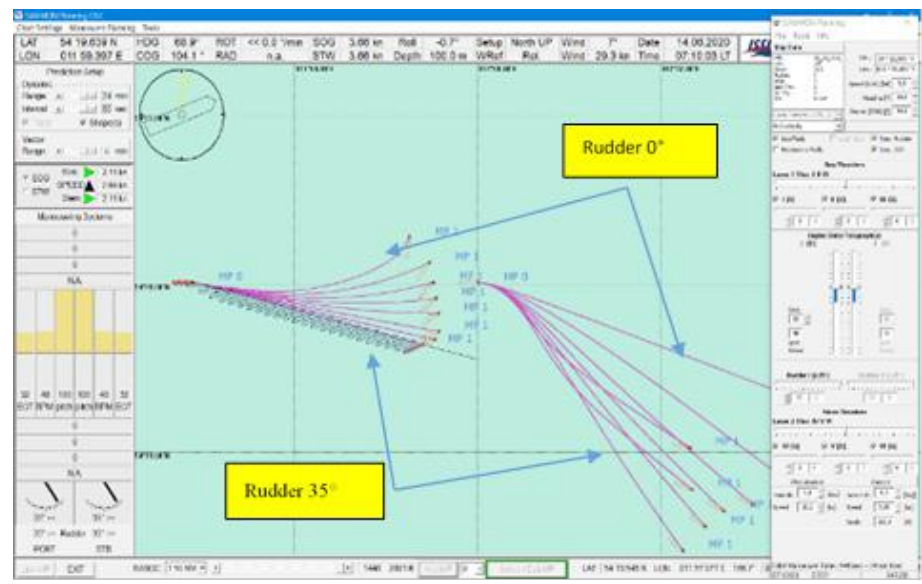

Figure 4. Ship tracks under wind for two cruise ships 
a. Left: Ship with centre of wind area aft (COSTA FORTUNA)

b. Right: Ship with centre of wind area fore (AIDAblu)

Wind: $0^{\circ}$, speed $30 \mathrm{kn}$; Ship: Initial speed $6 \mathrm{kn}$; Rudder: $0^{\circ}-35^{\circ}$ in $5^{\circ}$ steps, to Starboard.

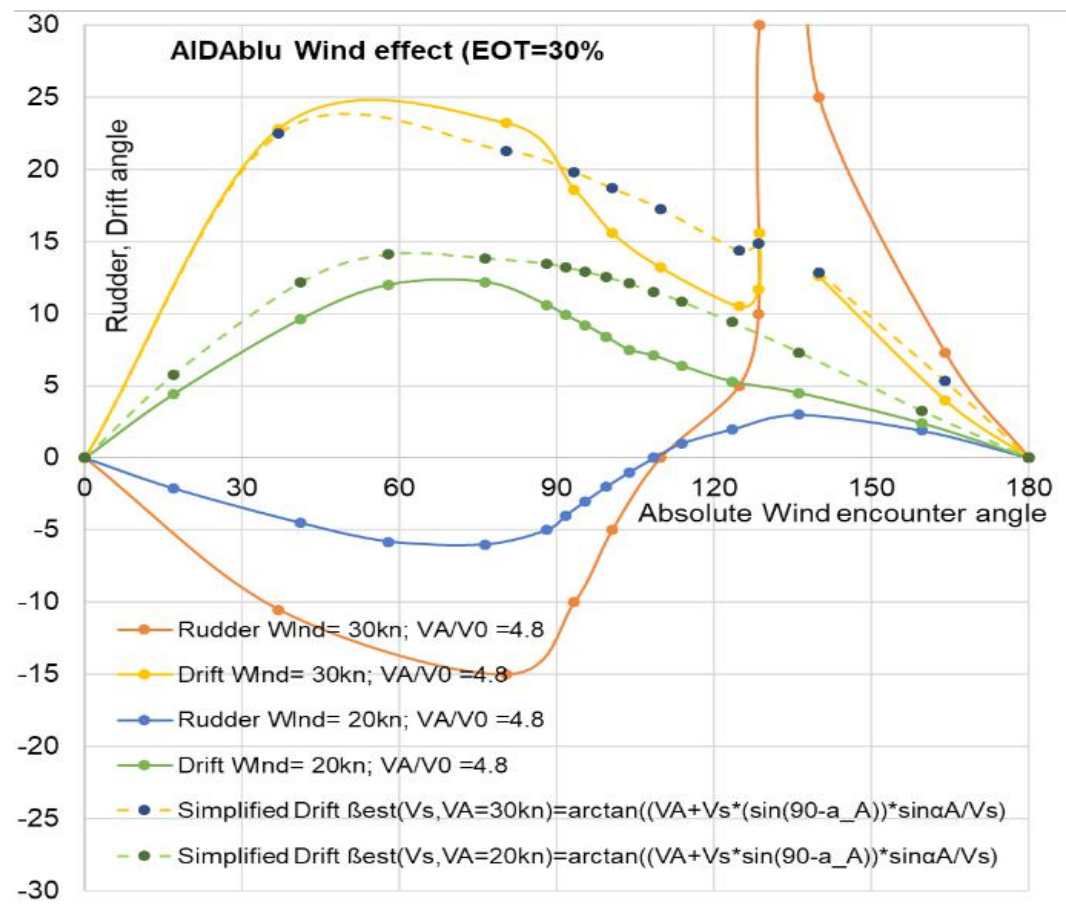

Figure 5. Rudder and drift angle versus absolute wind encounter angle for wind speeds of $20 \mathrm{kn}$ and $30 \mathrm{kn}$ (equilibrium on straight track, EOT=30\%, i.e. constant ship speed $\mathrm{V} 0=6.2 \mathrm{kn}$ )

a) Solid lines: Simulated data from Figure 4.

b) Dotted lines: simplified approach (2) for drift according to rule of thumb formula

\section{Planning and optimising manoeuvres for simulator training}

Task description for the exercise preparation - conventional briefing and new method

For preparing a simulator exercise e.g. for a port arrival, the trainee is commonly familiarised with the scenario, e.g. the port area, initial situation and environmental conditions. Normally this is done on a navigational chart, 
see e.g. Figure 7. In this sample exercise the ship should be brought through the fairway channel of Rostock Port from North, to be turned on the turning basin and then heading back through the channel to berth the ship with portside at the Passenger Pier. Manoeuvring sections would give a good overview on the different tasks / aims:

-1 st Section: the ship speed should slow down until around $3 \mathrm{kn}$, so she is ready to be turned.

- 2nd Section: the ship starts and performs the turning manoeuvre to be adjusted ready to go back in the fairway on opposite course.

- 3rd Section: the ship slows down to be finally stopped and brought to the berth.

In the conventional briefing and exercise preparation, only guessing and these rough indications of manoeuvring conditions can be used to develop a "manoeuvring plan" - specific manoeuvres and settings controls cannot be discussed in detail.

By means of the RAPIT Fast Time Simulation an individual exercise preparation with self-developed manoeuvring concepts would be possible:

- With the SAMMON Manoeuvre Planning Tool it would be easy and quick to develop a detailed Manoeuvring Plan and even the

- Improvement of the concept by several attempts or even "What-if" trials with that tool, e.g. for considering fuel consumptions and emissions.

In the following chapters a sample will be given to develop a manoeuvring plan for a ship with azimuth propellers according to the scenario above with two different strategies to use the PODs

The topic of this investigation was to find out the most effective manoeuvre with respect to energy / consumption and emissions for Port approaches. Two different strategies were used:

1. Conventional POD strategy, similar to twin propeller/rudder configuration, i.e. speed changes by changing POD revolutions and rudder angles

2. New POD strategy, with IN-OUT configuration, i.e. speed changes by turning both PODs inward or outward, or as tandem configuration (Figure 9)

In Figure 7 the plan for the 2. new POD strategy is shown, it looks nearly the same as the other variant 1 (therefore not extra shown here); the difference is in the details which will be discussed separately soon.

The two manoeuvres with different strategies were initially simulated with SAMMON Planning tool, resulting in two respective Manoeuvring Planning Files. Afterwards these files were taken as input to the SIMOPT / SIMDAT tool to be analysed in detail in order to assess the fuel consumption for further optimisation, if needed. 


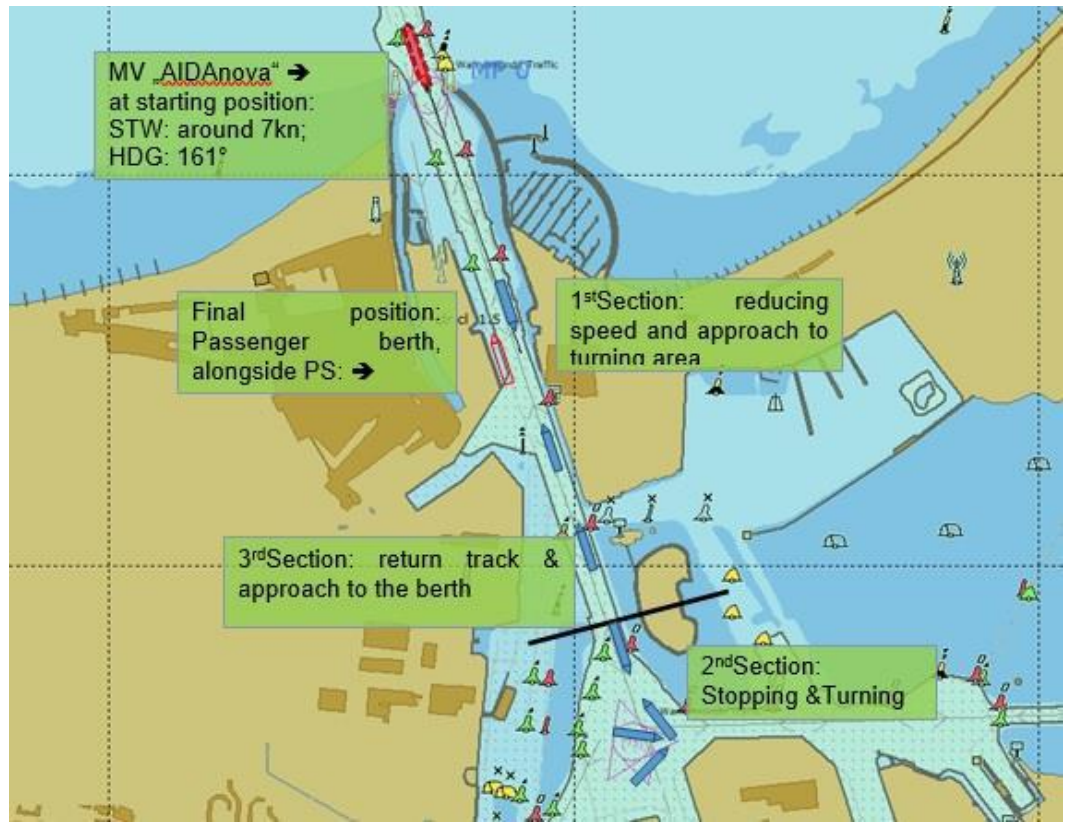

Figure 6. Sample for conventional briefing for a berthing scenarioin the Port of Rostock

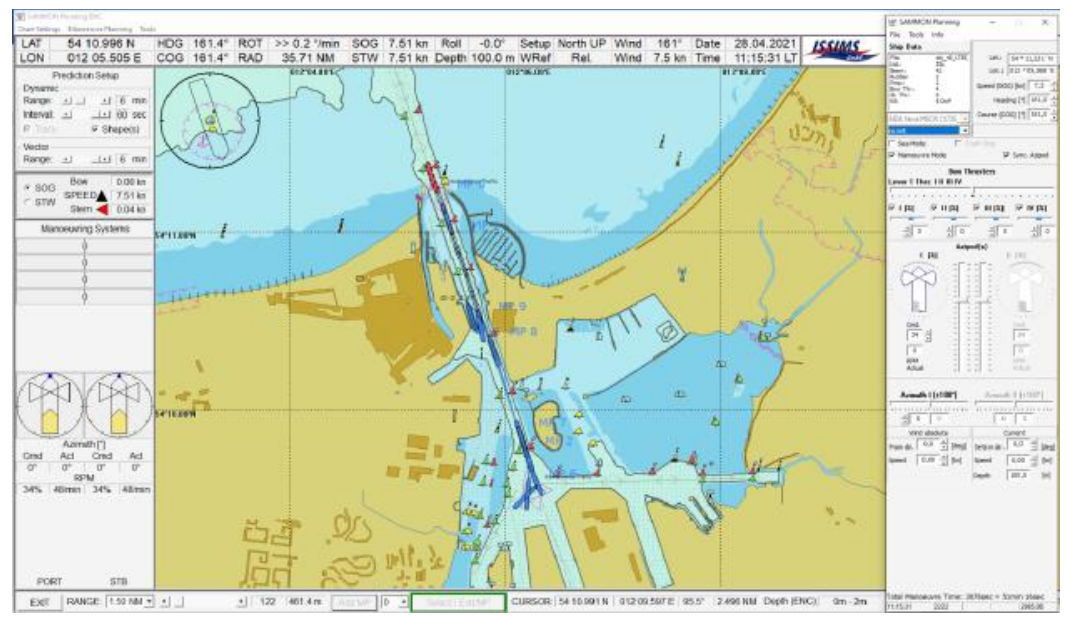

Figure 7. Sample with SAMMON Planning tool for arrival planning for Rostock port: Using new POD strategy with IN-OUT configuration, e.g. speed changes by turning PODs inward 


\section{Planning by means of Fast Time Simulation for two strategies}

With the new SAMMON Planning tool there is the chance for designing a Manoeuvre Plan with the specific control settings at specific positions called Manoeuvring Points MP. The following sample demonstrates how to make a full manoeuvring plan by means of the suitable control actions at the MPs.

In Figure 8 the initial position MP0 is to be seen where the ship was set in the centre of the fairway before entering the moles, the speed rate is initially EOT $=32 \%$, revealing a speed of $7.2 \mathrm{kn}$. The ship has already been moved by the time slider at the ENC bottom to set the next manoeuvring point MP1. There, the speed reduction manoeuvre is started:

- In the top figure it is done with reducing EOT to $10 \%$. The ship shapes of the predicted track ahead of the MP0 already show continuously reduced distances, i.e. the speed drops according to the new handle positions. And by moving the blue reference shape by means of the time slider to the new position at $360 \mathrm{sec}$, the speed there is only SOG $4.11 \mathrm{kn}$, to be seen in the top row. This is the same manoeuvre which typically would be done with a conventional twin screw vessel.

- In the bottom figure it is done with a typical POD manoeuvre by turning both pods inward to IN $30^{\circ}$. Now the thrust of the pods is not fully directed forward anymore, therefore the speed is dropping to $4.49 \mathrm{kn}$ after $360 \mathrm{sec}$.

This comparison of the speed drop after $360 \mathrm{sec}$ was only to show the effect of the two manoeuvres. The reference position may be moved further along the fairway to a suitable position where the next manoeuvre is necessary. A new Manoeuvring Point MP 2 can be set by pressing the button "Add MP" and then the focus is on the new MP 2 and so forth.
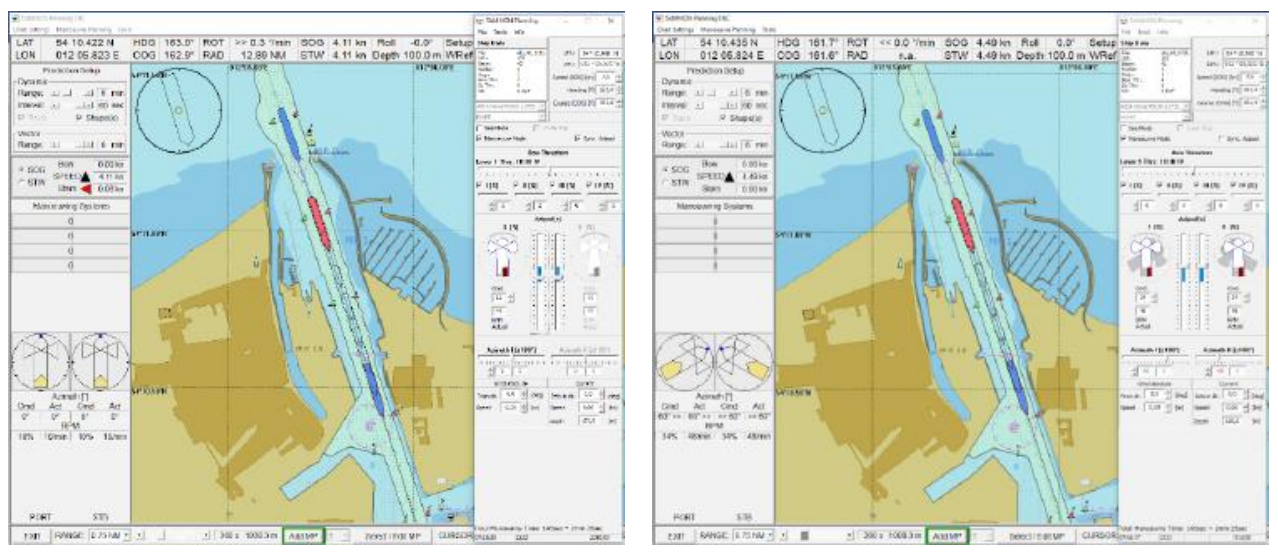

Figure 8. SAMMON planning interface: Starting position at MP0 and predicted shapes for the following slow down manoeuvre beginning at MP1: 
Top: in conventional way with reducing EOT to $10 \%$

Bottom: turning both pods inward IN $30^{\circ}$

In the next Figure 9 two different manoeuvres are shown to discuss the different turning strategies starting at position MP3:

In the top figure it is done in conventional way by stopping the port engine and turning the ship with the SB POD IN $100^{\circ}$ with transverse thrust like a rudder.

In the bottom figure the pods are used in a tandem position: PT POD IN120 and SB pod OUT60
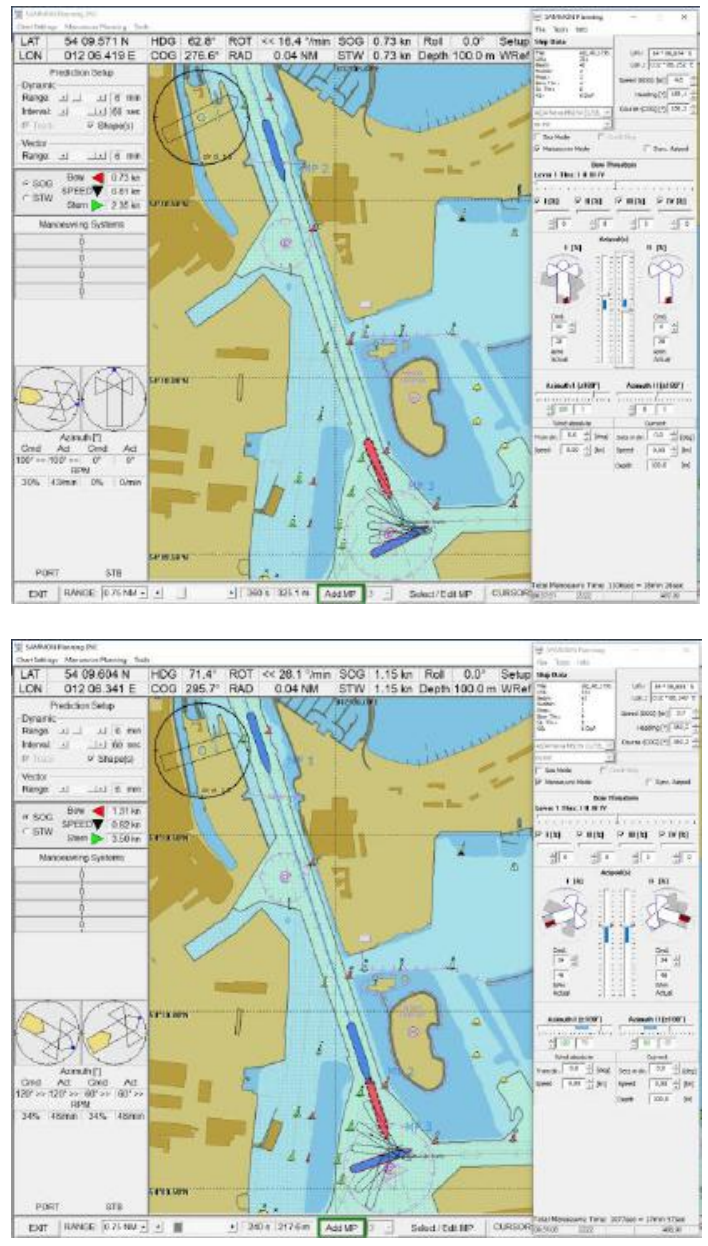

Figure 9. Two manoeuvres for different turning strategies starting at position MP3: 
- Top: conventional way by stopping the port engine PT POD EOT $0 \%$ and turning the ship with the SB POD to IN $100^{\circ}$ with EOT $30 \%$,

- Bottom: the pods are used in a tandem position: PT POD to IN $120^{\circ}$ and SB POD to OUT $60^{\circ}$, both with EOT $34 \%$

In the final phase of the manoeuvring plan the ship will be stopped short before the berth Figure 10. In the top figure the pods are used in a conventional way of stopping by reversing the pods to direct the thrust in astern direction. In the bottom the pods are used inward to create maximum resistance and on the same time adjusting the EOTs so that the ship is moving in the direction of the berth, together with the bow thrusters.
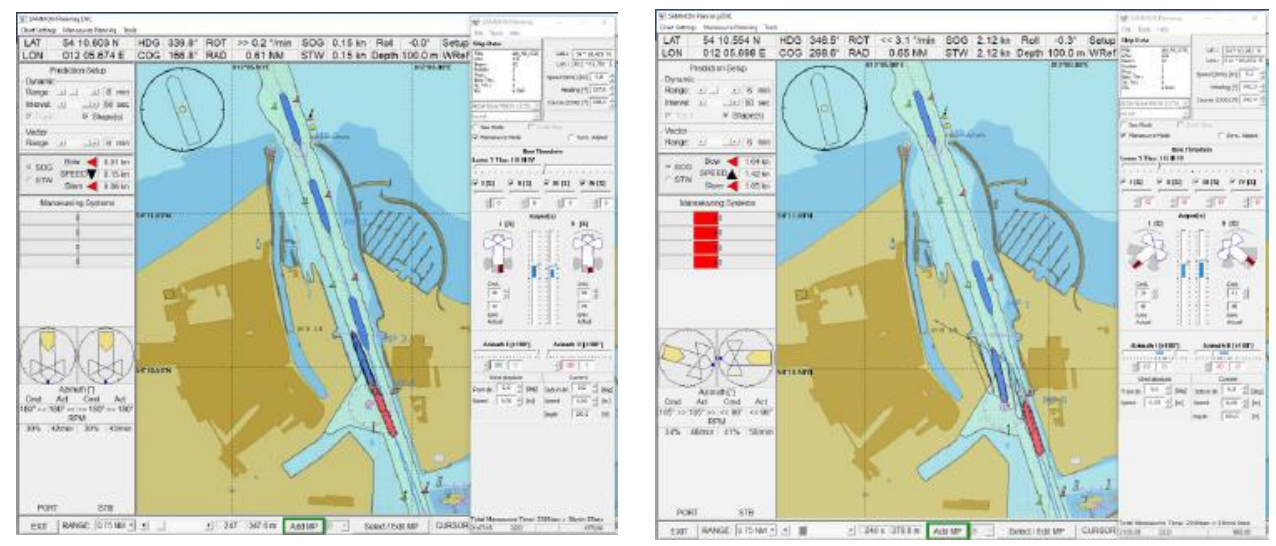

Figure 10. Two manoeuvres for different stopping strategies starting at position MP6:

- Top: turning the PODs astern $180^{\circ}$, keeping EOT 30\%,

- Bottom: the pods are used inward, PT POD to IN $105^{\circ}$, keeping EOT 34\%; and SB POD to OUT $60^{\circ}$, both with EOT $34 \%$

\section{Analysis of fuel consumption}

The question is now to decide which of the two strategies are better? For this reason, the fuel consumption was estimated for both versions by means of the SIMOPT $^{1 /} /$ SIMDAT software programs. In Figure 11 a comparison of controls for both manoeuvring strategies is made, it can be seen that the conventional strategy is using much lower revolutions but achieving nearly the same speed history.

The benefit is shown in Figure 12 comparing the consumption for both manoeuvring strategies for each manoeuvre separately (top) and as a cumulative sum of all the manoeuvres (bottom). It can be analysed with respect to consumption: 
a) Conventional POD- strategy: consumption ca. $3 \times 106$ g, i.e. ca $3 \mathrm{t}$

b) New POD-Inward strategy: ca. 9x106 g consumption, i.e. ca $9 \mathrm{t}$
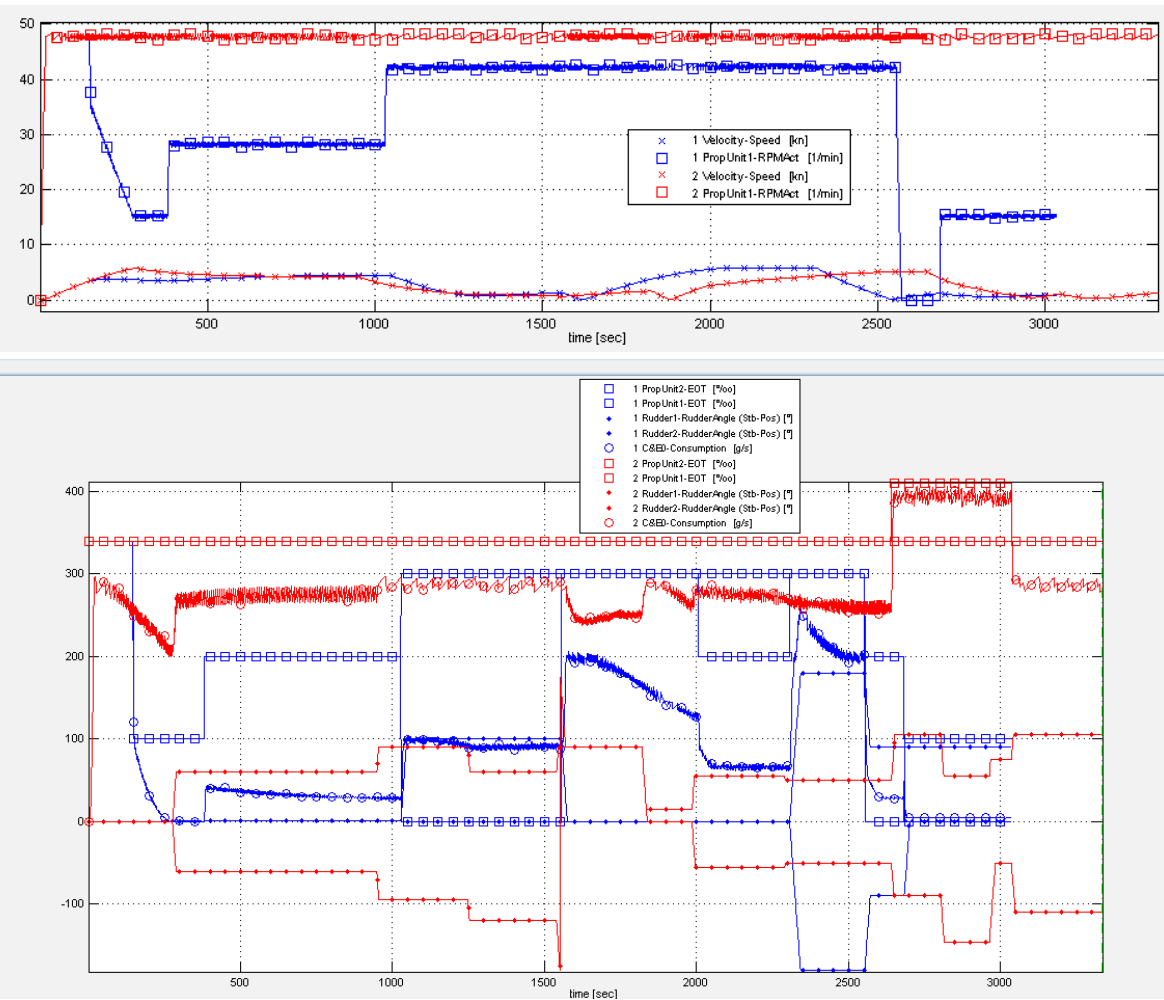

Figure 11. Comparison of controls for both manoeuvring strategies

- Top: Time history of speed and engine revolutions and

- Bottom: pod angles together with EOT and Revs

(Blue: Conventional POD strategy, speed changes by reducing POD revolutions -

Red: New POD strategy, speed changes by turning both PODs inward, shown as rudder units)

Therefore, the following conclusion can be made:

The conventional operation of PODs such as for a normal Twin Screw ship has only 30\% fuel consumption (and in analogy about 30\% emission of CO2), compared to the New operation: Operating the PODs against each other is a waste of energy and damage to the environment! 
Without any doubt the Inward strategy has a lot of advantages because the ship is much easier to control due to the immediately available steering forces when turning the pods for higher revolutions. But this should only be used if these high forces are really needed, e.g. for challenging external forces or complicated manoeuvres.
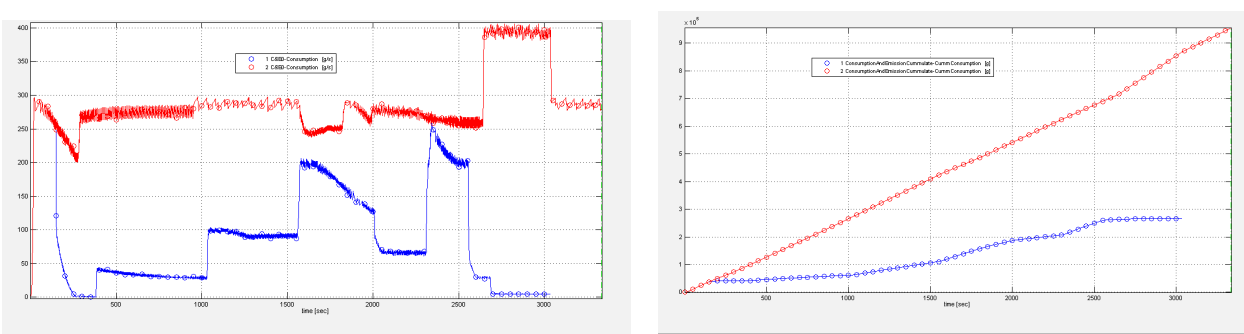

Figure 12. Comparison of consumption for both manoeuvring strategies

Top: Plot of consumption of separate manoeuvres

Bottom: Plot of cumulative consumption

(Blue: Conventional POD strategy, speed changes by reducing POD revolutions - Red: New POD strategy, speed changes by turning both PODs inward)

It should be mentioned that the models for fuel consumptions and emission used here are still simple and not precise enough the really calculate reliable results for the total values. However, they are a good estimation when comparing the relative data of consumption and emissions for two different manoeuvring strategies. For the time being the models are based on quasi-linear, steady state models, calculating of the fuel consumption is based on its calorific value, the propeller torque and its speed during the manoeuvres. New models for transient engine operation in non-steady operation are already under development, but needs much higher efforts for engine data and additional simulation parameters (Schaub et al. 2019; Schaub et al. 2021)

\section{Conclusions}

The examples have shown the benefits of using SAMMON Planning tool:

For lecturing it is much better to use samples by Fast-Time Manoeuvring Simulation instead of theoretical explanations; they are self-explanatory for specific ship behaviour and for the sample of wind drift two simple formulas could be developed to calculate the drift speed.

SAMMON allows even for preparing complete manoeuvring plans very fast - the same trials in a full mission SHS would take hours. It will be a great benefit to implement fuel consumption and emissions into the simulation: For the time being simple models were used to estimate and compare the fuel consumption for 
different manoeuvring strategies with each other. As a result, it could be shown that conventional operation of PODs for changing speed by changing revolution as for a normal Twin Screw ship has only $30 \%$ fuel consumption compared to the alternative new operation to control the speed by turning the PODs inward for speed reduction: Operating the PODs against each other is a waste of energy and damage to the environment!

SAMMON has proven already its benefits for lecturing and training and will have a great potential to increase efficiency and sustainability of manoeuvring also on-board.

Acknowledgments. Research results presented in this paper were partly achieved in "Euro-ZA Capacity building in the field of maritime education", a project funded by European Commission. The start-up company "Innovative Ship Simulation and Maritime Systems GmbH" (ISSIMS GmbH; www.issims-gmbh. com) is developing and maintaining the professional version of the SAMMON software tool.

\section{NOTES}

1. ISSIMS GmbH: SAMMON, Simulation Augmented Manoeuvring Design \& Monitoring System. Version 1.4, https://www.issims-gmbh.com/yoomla / products/simopt

2. ISSIMS GmbH - Marine Prediction Technology Youtube channel https://www. youtube.com/channel/UCR7yLtA5eqRUHNfQLXfgueA

\section{REFERENCES}

Benedict, K., Gluch, M., Fischer, S., Schaub, M., 2015. Innovative Manoeuvring Support by Simulation Augmented Methods - On-Board and from the Shore. In: Conference of the International Maritime Lecturers Association IMLA 23 [Durban /SA, 29 June to 3 July 2015].

Benedict, K., Gluch, M., Kirchhoff, M., Fischer, S., Schaub, M., 2016. Innovative Fast Time Simulation Tools For Briefing / Debriefing In: Advanced Ship Handling Simulator Training At Aida Cruises Rostock. In: 19th International Navigation Simulator Lecturers' Conference INSLC 19 [5 -8 September 2016 Cape Town /SA].

Schaub, M., Finger, G., Krüger, C., Tuschling, G., Baldauf, M., Benedict, K., 2019. Quantifying Fuel Consumption \& Emission in Ship Handling Simulation for Sustainable and Safe Operation in Harbour Areas. In: IAMUC2019 (Hg.): Proceedings of the International Association of Maritime Universities (IAMU) Conference. Tokyo. 
Benedict, K., Schaub, M., Tuschling, G., Baldauf, M., Gluch, M., Kirchhoff, M., 2021. Innovative SAMMON Teaching Method for Ship Handling: Element of the Project EURO-ZA Between South Africa and Europe. In: Bauk S., Ilčev S.D. (eds) The 1stInternational Conference on Maritime Education and Development. Springer, Cham. https://doi. org/10.1007/978-3-030-64088-0 1

Schaub, M., Benedict, K., Kirchhoff, M., 2021. Artificial Intelligence as a Practical Approach to Represent Complex Dynamic Relationships in Maritime Navigation. In: Bauk S., Ilčev S.D. (eds) The $1^{\text {st }}$ International Conference on Maritime Education and Development. Springer, Cham. https://doi.org/10.1007/978-3-030-64088-0_2

$\triangle$ Knud Benedict

Department of Maritime Studies Warnemuende Institute ISSIMS

Wismar, Germany E-mail:knud.benedict@hs-wismar.de

$\triangle$ Michèle Schaub

Department of Maritime Studies Warnemuende Institute ISSIMS

Wismar, Germany

$\triangle$ Michael Baldauf

https://orcid.org/0000-0002-7333-0348 Department of Maritime Studies Warnemuende Institute ISSIMS Wismar, Germany

$\triangle$ Michael Gluch

Department of Maritime Studies Warnemuende Institute ISSIMS

Wismar, Germany

$\triangle$ Matthias Kirchhoff ISSIMS GmbH Rostock, Germany E-mail: mkirchhoff@issim-gmbh.de

\section{Caspar Krüger} ISSIMS GmbH Rostock, Germany 